\title{
BRAIN-LIKE FUNCTOR CONTROL MACHINE FOR GENERAL HUMANOID BIODYNAMICS
}

\author{
VLADIMIR IVANCEVIC AND NICHOLAS BEAGLEY
}

Received 7 June 2004 and in revised form 27 February 2005

A novel, brain-like, hierarchical (affine-neuro-fuzzy-topological) control for biomechanically realistic humanoid-robot biodynamics (HB), formulated previously in $[15,16]$, is proposed in the form of a tensor-invariant, "meta-cybernetic" functor machine. It represents a physiologically inspired, three-level, nonlinear feedback controller of muscularlike joint actuators. On the spinal level, nominal joint-trajectory tracking is formulated as an affine Hamiltonian control system, resembling the spinal (autogenetic-reflex) "motor servo." On the cerebellar level, a feedback-control map is proposed in the form of selforganized, oscillatory, neurodynamical system, resembling the associative interaction of excitatory granule cells and inhibitory Purkinje cells. On the cortical level, a topological "hyper-joystick" command space is formulated with a fuzzy-logic feedback-control map defined on it, resembling the regulation of locomotor conditioned reflexes. Finally, both the cerebellar and the cortical control systems are extended to provide translational force control for moving 6-degree-of-freedom chains of inverse kinematics.

\section{Introduction}

Traditional hierarchical robot control (see, e.g., [31]) consists of three control levels: the executive (bottom) level performs tracking of nominal trajectories in internal joint coordinates, the strategic (top) level performs "planning" of the trajectories of an end-effector in external Cartesian coordinates, and the tactical (middle) level connects other two levels by means of inverse kinematics.

The modern version of the hierarchical robot control (see, e.g., [20]) includes decision making performed by the neuro-fuzzy(-genetic) classifier to adapt the (manipulator) control to dynamically changing environment.

The so-called "intelligent" approach to robot control typically represents a form of function approximation, which is itself based on a combination of neuro-fuzzy-genetic computations (see, e.g., [7]).

In addition, many special issues and workshops focusing on physiological models for robot control reflect the increased attention for the development of cerebellar models 
$[30,2,3,4,25,26]$ for learning robot control with functional decomposition. Here, the main result could be formulated as the cerebellum is more than just the function approximator.

In this paper, we introduce an alternative approach for humanoid control, emphasizing the role of muscle-like actuators. We propose a new, physiologically based, tensorinvariant, hierarchical force control (FC) for the $\mathrm{HB}$, formulated previously in $[14,15$, $16,17,18,19]$. We consider the muscle-like covariant driving torques, that is, one-forms $F_{i}=F_{i}(t, q, p)$, which are dependent on time $t$, joint angles $q=q(t)$, and canonical angular momenta $p=p(t)$, as the most important component of humanoid motion (this is based on the fact of extremely high degree of the natural muscular redundancy: the human body, which is an everlasting inspiration for humanoid robots, for its motion uses a synergetic action of approximately 640 skeletal muscles); therefore we propose the sophisticated hierarchical system for the subtle $F_{i}$-control corresponding to the spinal, the cerebellar, and the cortical levels of human motor control. $F_{i}$ are first set up as testing input signals to $\mathrm{HB}$, and then covariantly updated as feedback one-forms $\boldsymbol{u}_{i}$ on each FC level. On the spinal FC level, the nominal joint-trajectory tracking is proposed in the form of affine Hamiltonian control; here the driving torques are given corrections by spinal-reflex controls. On the cerebellar FC level, the relation is established between canonical joint coordinates $q^{i}, p_{i}$ and gradient neural-image coordinates $x^{i}, y_{i}$, representing bidirectional, self-organizing, associative memory machine; here the driving torques are given the cerebellar corrections. On the cortical FC level, the topological "hyper-joystick" is proposed as the central FC command-space selector, with the fuzzylogic feedback-control map defined on it, giving the cortical corrections to the driving torques.

The model of the spinal FC level, formulated here, resembles an autogenetic motor servo [9] acting on the spinal-reflex level of the human locomotor control. (Voluntary contraction force $\Phi$ of human skeletal muscle is reflexly excited (positive feedback $+\Phi^{-1}$ ) by responses of its spindle receptors to stretch and is reflexly inhibited (negative feedback $-\Phi^{-1}$ ) by responses of its Golgi tendon organs to contract. Stretch and unloading reflexes are mediated by combined actions of several autogenetic neural pathways, forming the so-called "motor servo." The term "autogenetic" means that the stimulus excites receptors located in the same muscle that is the target of the reflex response. The most important of these muscle receptors are the primary and secondary endings in muscle spindles, sensitive to length change-positive length feedback $+\Phi^{-1}$, and the Golgi tendon organs, sensitive to contractile force-negative force feedback $-\Phi^{-1}$. The gain $G$ of the length feedback $+\Phi^{-1}$ can be expressed as the positional stiffness (the ratio $G \approx S=d \Phi / d x$ of the force- $\Phi$ change to the length- $x$ change) of the muscle system. The greater the stiffness $S$, the less the muscle will be disturbed by a change in load and the more reliable the performance of the muscle system in executing controlled changes in length $+\Phi^{-1}$. The autogenetic circuits $+\Phi^{-1}$ and $-\Phi^{-1}$ appear to function as servoregulatory loops that convey continuously graded amounts of excitation and inhibition to the large (alpha) skeletomotor neurons. Small ( $\mathrm{gamma}$ ) fusimotor neurons innervate the contractile poles of muscle spindles and function to modulate spindle-receptor discharge.) The model of the cerebellar FC level, formulated here, mimics the self-organizing, associative function 
of the excitatory granule cells and the inhibitory Purkinje cells of the cerebellum [10]. The model of the cortical FC level, presented in this paper, mimics the integral synergistic regulation of (loco)motor conditioned reflexes [10].

Finally, both cerebellar control systems can be easily extended to provide translational force control for moving 6-degree-of-freedom inverse kinematics chains.

It is our view that the extremely high order of the driving force redundancy in the biomechanically realistic HB justifies the formulation of the three-level force control functor system.

Computer-algebra implementation of all three FC levels of $\mathrm{HB}$ is provided in the appendix.

\section{Functor control machine}

In this section, we define the functor control machine (see $[14,15,18,19])$, for the learning control with functional decomposition, by a two-step generalization of Kalman's theory of linear MIMO feedback systems. The first generalization puts Kalman's theory into the pair of mutually dual linear categories Vect and Vect* of vector spaces and linear operators, with a "loop functor" representing the closed-loop control, thus formulating the unique, categorical formalism valid both for the discrete, and for the continual MIMO systems.

We start with the unique, feedforward continual-sequential state equation

$$
\dot{x}(t+1)=A x(t)+B u(t), \quad y(t)=C x(t)
$$

where (as usual) the overdot denotes the time derivative, and the finite-dimensional vector spaces of states $X \ni x$, inputs $U \ni u$, and outputs $Y \ni y$ have the corresponding linear operators, respectively, $A: X \rightarrow X, B: U \rightarrow X$, and $C: X \rightarrow Y$. The modular system theory comprises the system dynamics, given by a pair $(X, A)$, together with a reachability map $e: U \rightarrow X$ of the pair $(B, A)$, and an observability map $m: X \rightarrow Y$ of the pair $(A, C)$. If the reachability map $e$ is a surjection, the system dynamics $(X, A)$ is called reachable; if the observability map $m$ is an injection, the system dynamics $(X, A)$ is called $o b$ servable. If the system dynamics $(X, A)$ is both reachable and observable, a composition $r=m \circ e: U \rightarrow Y$ defines the total system's response, which is given by solution of (2.1). If the unique solution to the continual-sequential state equation exists, it gives the answer to the (minimal) realization problem: find the system $S$ that realizes the given response $r=m \circ e: U \rightarrow Y$ (in the smallest number of discrete states and in the shortest time).

The inverse map $r^{-1}=e^{-1} \circ m^{-1}: Y \rightarrow U$ of the total system's response $r: U \rightarrow Y$ defines the linear feedback operator $K: Y \rightarrow U$, given by standard feedback equation

$$
u(t)=K y(t)
$$

In categorical language, see $[15,17,18,19]$, the feedforward system dynamics in the category Vect is a pair $(X, A)$, where $X \in \mathrm{Ob}$ (Vect) is an object in Vect and $A: X \rightarrow$ $X \in \operatorname{Mor}($ Vect) is a Vect-morphism. A feedforward decomposable system in Vect is 
such a sextuple $S \equiv(X, A, U, B, Y, C)$ that $(X, A)$ is the system dynamics in Vect, a Vectmorphism $B: U \rightarrow X$ is an input map, and a Vect-morphism $C: X \rightarrow Y$ is an output map. Any object in Vect is characterized by mutually dual (recall that in categorical language, duality means reversing the (arrows of) morphisms; the knowledge of one of the two mutually dual terms automatically implies the knowledge of the other) notions of its degree (a number of its input morphisms) and its codegree (a number of its output morphisms). Similarly, any decomposable system $S$ in Vect has a reachability map given by an epimorphism $e=A \circ B: U \rightarrow X$ and its dual observability map given by a monomorphism $m=C \circ A: X \rightarrow Y$; their composition $r=m \circ e: U \rightarrow Y$ in Mor(Vect) defines the total system's response in Vect given by the unique solution of the continual-sequential state equation (2.1).

The dual of the total system's response, defined by the feedback equation (2.2), is the feedback morphism $K=e^{-1} \circ m^{-1}: Y \rightarrow U$ belonging to the dual category Vect* .

In this way, the linear, closed-loop, continual-sequential MIMO system (2.1) and (2.2) represents the linear iterative loop functor $\mathscr{L}$ : Vect $\Rightarrow$ Vect* .

Our second generalization represents a natural system process $\Xi[\mathscr{L}]$ that transforms the linear loop functor $\mathscr{L}:$ Vect $\Rightarrow$ Vect $^{*}$ into the nonlinear loop functor $\mathcal{N} \mathscr{L}: \mathscr{C} \mathscr{A} \mathscr{T} \Rightarrow$ $\mathscr{C} \mathscr{A} \mathscr{T}^{*}$ between two mutually dual nonlinear categories $\mathscr{C} \mathscr{A} \mathscr{T}$ and $\mathscr{C} \mathscr{A} \mathscr{T}^{*}$. We apply the natural process $\Xi$ separately

(1) on the feedforward decomposable system $S \equiv(X, A, U, B, Y, C)$ in Vect,

(2) on the feedback morphism $K=e^{-1} \circ m^{-1}: Y \rightarrow U$ in Vect*.

Under the action of the natural process $\Xi$, the linear feedforward system dynamics $(X, A)$ in Vect transforms into a nonlinear feedforward $\Xi$-dynamics $(\Xi[X], \Xi[A])$ in $\mathscr{C} A \mathcal{T}$, represented by a nonlinear feedforward decomposable system $\Xi[S] \equiv(\Xi[X], \Xi[A]$, $\Xi[U], \Xi[B], \Xi[Y], \Xi[C])$.

The reachability map transforms into the input process $\Xi[e]=\Xi[A] \circ \Xi[B]: \Xi[U] \rightarrow$ $\Xi[X]$, while its dual observability map transforms into the output process $\Xi[m]=\Xi[C] \circ$ $\Xi[A]: \Xi[X] \rightarrow \Xi[Y]$. In this way, the total response of the linear system $r=m \circ e: U \rightarrow Y$ in Mor(Vect) transforms into the nonlinear system behavior $\Xi[r]=\Xi[m] \circ \Xi[e]: \Xi[U] \rightarrow$ $\Xi[Y]$ in $\operatorname{Mor}(\mathscr{C A F})$. Obviously, $\Xi[r]$, if exists, is given by a nonlinear $\Xi$-transform of the linear state equation (2.1).

Analogously, the linear feedback morphism $K=e^{-1} \circ m^{-1}: Y \rightarrow U$ in Mor(Vect*) transforms into the nonlinear feedback morphism $\Xi[K]=\Xi\left[e^{-1}\right] \circ \Xi\left[m^{-1}\right]: \Xi[Y] \rightarrow$ $\Xi[U]$ in $\operatorname{Mor}\left(\mathscr{C} \mathscr{A} \mathscr{T}^{*}\right)$.

In this way, the natural system process $\Xi: \mathscr{L} \Rightarrow \mathcal{N} \mathscr{L}$ is established. This means that the nonlinear loop functor $L=\Xi[\mathscr{L}]: \mathscr{C} \mathscr{A} \mathcal{T} \Rightarrow \mathscr{C A} \mathcal{F}^{*}$ is defined out of the linear, closedloop, continual-sequential MIMO system (2.1) and (2.2).

The purpose of this paper is to formulate the nonlinear loop functor $L=\Xi[\mathscr{L}]$ : $\mathscr{C} \mathscr{A T} \Rightarrow \mathscr{C} A \mathscr{T}^{*}$ for various hierarchical levels of muscular-like FC.

\section{Generalized Hamiltonian HB plant}

Generalized HB plant has been previously developed in $[14,15,17,18,19]$. For the sake of continuity of the present paper, in this section we briefly recapitulate the main points. 


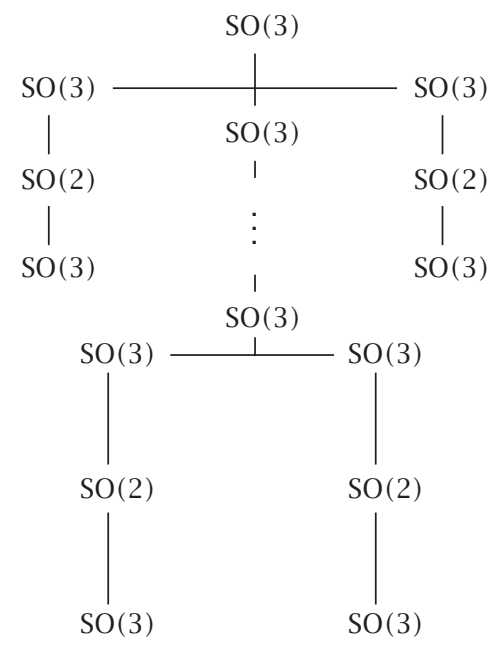

Figure 3.1. HB configuration manifold defined as an anthropomorphic product tree of the constrained rotational Lie groups.

Kinematics of an $n$-segment humanoid chain is usually defined as a map between external (usually, end-effector) coordinates $x^{r}(r=1, \ldots, n)$ and internal (joint) coordinates $q^{i}(i=1, \ldots, N)$. The forward kinematics is defined as a nonlinear map $x^{r}=$ $x^{r}\left(q^{i}\right)$ with corresponding linear vector functions $d x^{r}=\partial x^{r} / \partial q^{i} \cdot d q^{i}$ of differentials, and $\dot{x}^{r}=\partial x^{r} / \partial q^{i} \cdot \dot{q}^{i}$ of velocities. (Here and subsequently the summation convention over repeated indices is understood.) When the rank of the configuration-dependent Jacobian matrix $J \equiv \partial x^{r} / \partial q^{i}$ is less than $n$, the kinematic singularities occur; the onset of this condition could be detected by the manipulability measure [34]. Inverse kinematics (IK) is defined conversely by a nonlinear map $q^{i}=q^{i}\left(x^{r}\right)$ with corresponding linear vector functions $d q^{i}=\partial q^{i} / \partial x^{r} \cdot d x^{r}$ of differentials and $\dot{q}^{i}=\partial q^{i} / \partial x^{r} \cdot \dot{x}^{r}$ of velocities. Again, in the case of redundancy $(n<N)$, the inverse kinematic problem admits infinite solutions; often the pseudoinverse configuration control is used instead [28]: $\dot{q}^{i}=J^{*} \cdot \dot{x}^{r}$, where $J^{*}=J^{T}\left(J J^{T}\right)^{-1}$ denotes the Moore-Penrose pseudoinverse of the Jacobian matrix $J$.

The joint angles $q^{i}(i=1, \ldots, N \equiv \mathrm{DOF})$ in HB constitute a smooth $\left(C^{\infty}\right)$ configuration manifold $M^{N}$, defined as a direct product of joint rotational Lie groups $\mathrm{SO}(3) \times$ $\mathrm{SO}(2) \times \mathrm{SO}(3) \times \ldots$ for all joints (Figure 3.1). Uniaxial, "hinge" joints represent constrained, classical, rotational Lie groups $\mathrm{SO}(2)_{\text {cnstr }}^{i}$, parameterized by constrained angles $q_{\text {cnstr }}^{i} \equiv q^{i} \in\left[q_{\min }^{i}, q_{\max }^{i}\right]$. (In the following text, the subscript "cnstr" will be omitted, for the sake of simplicity.) Three-axial, "ball-and-socket" joints represent constrained rotational groups $\mathrm{SO}(3)^{i}$, usually parameterized by constrained Euler angles $q_{1,2,3}^{i}=$ $\{\phi, \psi, \theta\}^{i}$.

If we apply the functor Lie on the category $\cdot\left[\mathrm{SO}(n)^{i}\right]$ (for $n=2,3$ and $i=1, \ldots, N$ ) of rotational Lie groups $\mathrm{SO}(n)^{i}$, we obtain the category $\cdot\left[\operatorname{so}(n)_{i}\right]$ of corresponding tangent Lie algebras so $(n)_{i}($ see $[14,15,18,19])$. If we further apply the functor Dual ${ }_{G}$ to the 
category $\left[\operatorname{so}(n)_{i}\right]$, we obtain the category $*^{*}\left[\operatorname{so}(n)_{i}^{*}\right]$ of cotangent, or, canonical Lie algebras $\operatorname{so}(n)_{i}^{*}$ (and their homomorphisms). To go directly from ${ }^{\bullet}\left[\mathrm{SO}(n)^{i}\right]$ to ${ }^{*}\left[\operatorname{so}(n)_{i}^{*}\right]$, we use the canonical functor Can.

Both the tangent algebras $\operatorname{so}(n)_{i}$ and the cotangent algebras so $(n)_{i}^{*}$ contain infinitesimal group generators: angular velocities $\dot{q}^{i}=\dot{q}^{\phi_{i}}$ in the first case, and canonical angular momenta $p_{i}=p_{\phi_{i}}$ in the second case. As Lie group generators, both the angular velocities and the angular momenta satisfy the commutation relations $\left[\dot{q}^{\phi_{i}}, \dot{q}^{\psi_{i}}\right]=\epsilon_{\theta}^{\phi \psi} \dot{q}^{\theta_{i}}$ and $\left[p_{\phi_{i}}, p_{\psi_{i}}\right]=\epsilon_{\phi \psi}^{\theta} p_{\theta_{i}}$, respectively, where the structure constants $\epsilon_{\theta}^{\phi \psi}$ and $\epsilon_{\phi \psi}^{\theta}$ constitute the totally antisymmetric third-order tensors.

Note that the parameterization of rotation is the subject of continuous research and development in many theoretical and applied fields of mechanics, such as rigid body, structural, and multibody dynamics, robotics, spacecraft attitude dynamics, navigation, image processing, and so forth. Besides Euler angles used in this paper, also Euler parameters, Cayley-Rodrigues parameters, exponential map of rotation, as well as quaternions have been classically used for more efficient parameterizations of the rotation group $\mathrm{SO}(3)$ (for a complete discussion on the classical attitude representations, see [5, 22, 27, 29]). In addition, a modern vectorial parameterization of finite rotations encompasses the mentioned earlier developments as well as Gibbs, Wiener, and Milenkovic parameterizations $[1,23]$.

Now, dissipative, driven $\delta$-Hamiltonian HB system on the configuration manifold $M^{N}$ is, in local canonical-symplectic coordinates $q^{i}, p_{i} \in U_{p}$ on the momentum phase-space manifold $T^{*} M^{N}$, given by autonomous equations

$$
\begin{gathered}
\dot{q}^{i}=\frac{\partial H_{0}}{\partial p_{i}}+\frac{\partial R}{\partial p_{i}}, \\
\dot{p}_{i}=F_{i}-\frac{\partial H_{0}}{\partial q^{i}}+\frac{\partial R}{\partial q^{i}}, \\
q^{i}(0)=q_{0}^{i}, \quad p_{i}(0)=p_{i}^{0} \quad(i=1, \ldots, N)
\end{gathered}
$$

including contravariant equation (3.1), the velocity vector field, and covariant equation (3.2), the force one-form, together with initial joint angles $q_{0}^{i}$ and momenta $p_{i}^{0}$. Here the physical Hamiltonian function $H_{0}: T^{*} M^{N} \rightarrow \mathbb{R}$ represents the humanoid total energy function, in local canonical coordinates $q^{i}, p_{i} \in U_{p}$ on $T^{*} M^{N}$ given by

$$
H_{0}(q, p)=\frac{1}{2} g^{i j} p_{i} p_{j}+V(q)
$$

where $g^{i j}=g^{i j}(q, m)$ denotes the contravariant material metric tensor (associated with Riemannian metrics $g: T M^{N} \rightarrow \mathbb{R}$ on $M^{N}$ ) relating internal and external HB coordinates (i.e., joint angles $q^{i}$ and Cartesian coordinates $x^{r}$, respectively), and including $n$ segmental 
masses $m_{\mu}$

$$
g^{i j}(q, m)=\sum_{\mu=1}^{n} m_{\mu} \delta_{r s} \frac{\partial q^{i}}{\partial x^{r}} \frac{\partial q^{j}}{\partial x^{s}}
$$

$R=R(q, p)$ denotes the Rayleigh nonlinear (usually biquadratic) dissipation function, and the one-forms $F_{i}=F_{i}(t, q, p, u)$ are generalized driving torques, depending on joint angles and momenta, as well as on $u_{i}=u_{i}(t, q, p)$, corrections from all control levels. Biomechanically speaking, the torques $F_{i}$ resemble neuro-muscular excitation and contraction dynamics of equivalent antagonistic muscular pairs in the ith joint (see $[15,17$, $18,19])$.

Neuro-muscular dynamics, giving the driving torques $F_{i}=F_{i}(t, q, p)$ for $\mathrm{HB}$, should describe the internal excitation and contraction dynamics $[14,15,17,18,19]$ of equivalent muscular actuators, anatomically represented by the resulting action of antagonistic muscle pairs for each uniaxial (i.e., $\mathrm{SO}(2)$ ) humanoid joint. We attempt herein to describe the equivalent muscular dynamics in the simplest possible way (e.g., Hatze used 51 nonlinear differential equations of the first order to derive his, arguably most elaborate, myocybernetic model [6]), and yet to include the main excitation and contraction relations.

(a) Excitation dynamics can be in the simplest way described by Gaussian bell-shaped torque-time relation $F_{i}^{\text {Gauss }}=F_{i}^{0} e^{-\left(t-\mu_{i}\right)^{2} /\left(2 \sigma_{i}^{2}\right)}$, where $F_{i}^{0}$ denote the maximal isometric muscular torques applied at the $i$ th joint $(i=1, \ldots, N)$, while $\mu_{i}$ and $\sigma_{i}$ denote, respectively, the time means and standard deviations of individual muscular contractions. This is a rotational joint-form approximation for the impulse plus transient response of Wilkie's muscular active-state element, defined by an RC circuit-like equation [33] $\dot{x}+\beta x=\beta S A$, $x(0)=0,0<S<1$, where $x=x(t)$ represents the active state of the muscle, $\beta$ denotes the element gain, $A$ corresponds to the maximum tension the element can develop, and $S=S(r)$ is the "desired" active state as a function of motor unit stimulus rate $r$.

(b) Contraction dynamics has classically been described by Hill's hyperbolic forcevelocity relation [8], which we propose here in the rotational $(q, p)$ form $F_{i}^{\text {Hill }}=\left(F_{i}^{0} b_{i}-\right.$ $\left.a_{i} p_{i}\right) /\left(p_{i}-b_{i}\right), i=1, \ldots, N$, where $a_{i}$ (having dimension of torque) and $b_{i}$ (having dimension of momentum) denote the rotational Hill parameters (see [14]) corresponding to the energy dissipated during the contraction and the phosphagenic energy conversion rate, respectively.

Therefore, we can describe the excitation/contraction dynamics for the $i$ th equivalent muscle-joint actuator, that is, antagonistic muscle pair (e.g., flexion/extension in the $i$ th joint) by the simple impulse-hyperbolic product relation $F_{i}(t, q, p)=F_{i}^{\text {Gauss }} \times F_{i}^{\text {Hill }}, i=$ $1, \ldots, N$.

As an illustrative example of the $\mathrm{HB}$ application, we have developed a standalone full-spine dynamics and crash simulator, including 25 constrained ball-end-socket joints with the total of 75 rotational degrees of freedom, implementing the spinal control level (see Figure 3.2 and Section 4) (developed in Mathematica (see the appendix) and subsequently implemented in Delphi environment for MS Windows). The general HB configuration manifold $M^{N}$ is now reduced to the 75 -dimensional torus $T^{75}$ (see (6.2) below) 


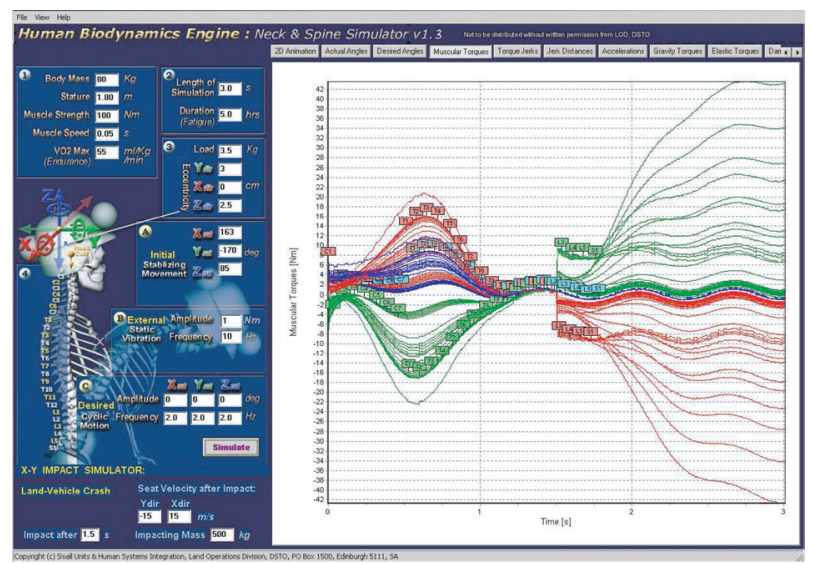

Figure 3.2. The full-spine dynamics and crash HB simulator, implementing the spinal control level: interface and a sample output of muscular torques. Simulating the exponentially stabilizing movement (returning to the normal vertical posture from the user-specified head displacement defined by the three initial angles around $X, Y$, and $Z$ axes), followed by a moderate impact (car crash) at 1.5 seconds after the simulation start.

and the momentum phase-space manifold corresponds to its 150-dimensional cotangent bundle $T^{*} T^{75}$. In this case, the canonical velocity and force (3.1) and (3.2) obtain relatively simple expanded forms

$$
\begin{aligned}
\dot{q}^{i}= & p_{i}\left\{\left[J_{i}\right]^{-1}+\left[m_{i}\left(\sum_{j=1}^{i} L_{j} \cos q^{j}\right)^{2}\right]^{-1}\right\}+\frac{\partial R}{\partial p_{i}}, \\
\dot{p}_{i}= & F_{i}\left(t, q^{i}, p_{i}\right)-g \sum_{j=i}^{76-i} L_{j} m_{j} \sin q^{j} \\
& -\sum_{j=i}^{76-i} L_{j} \sin q^{j} p_{i} p_{j}\left[m_{i}\left(\sum_{k=1}^{i} L_{k} \cos q^{k}\right)^{3}\right]^{-1}+\frac{\partial R}{\partial q^{i}}, \\
q^{i}(0)= & q_{0}^{i}, \quad p_{i}(0)=p_{i}^{0} \quad(i=1, \ldots, 75) .
\end{aligned}
$$

Here Ls, $m s$, and Js denote the segment lengths, masses, and inertia moments, respectively, and $g$ is the common gravity constant. In the contravariant velocity (3.6), the terms denote rotational velocities, translational velocities, and velocity dampings for the $i$ th joint, respectively, whereas the covariant force (3.7) contains terms of muscular excitation/contraction actuator torques $F_{i}=F_{i}\left(t, q^{i}, p_{i}\right)$, and passive-external torques (gravitational, Coriolis, centrifugal, and joint dampings, respectively).

Fuzzification of the crisp $\delta$-Hamiltonian HB system (3.1), (3.2), and (3.3) gives the fuzzified $\mu$-Hamiltonian HB system $[15,19]$, namely, $\delta$-Hamiltonian HB system with 
fuzzy system numbers (SN)

$$
\begin{gathered}
\dot{q}^{i}=\frac{\partial H_{0}\left(q, p, \sigma_{\mu}\right)}{\partial p_{i}}+\frac{\partial R}{\partial p_{i}}, \\
\dot{p}_{i}=\bar{F}_{i}-\frac{\partial H_{0}\left(q, p, \sigma_{\mu}\right)}{\partial q^{i}}+\frac{\partial R}{\partial q^{i}}, \\
q^{i}(0)=\bar{q}_{0}^{i}, \quad p_{i}(0)=\bar{p}_{i}^{0} \quad(i=1, \ldots, N),
\end{gathered}
$$

where $\{\sigma\}_{\mu}$ (with $\mu \geq 1$ ) denote fuzzy sets of conservative parameters (segment lengths, masses, and moments of inertia), dissipative joint dampings, and actuator parameters (amplitudes and frequencies), while the bar $(\cdot)$ over a variable $(\cdot)$ denotes the corresponding fuzzified variable.

Particularly, in the spinal HB simulator, the fuzzified $\mu$-Hamiltonian HB system (3.9) becomes

$$
\begin{aligned}
\dot{q}^{i}= & p_{i}\left\{\left[\bar{J}_{i}\right]^{-1}+\left[\bar{m}_{i}\left(\sum_{j=1}^{i} \bar{L}_{j} \cos q^{j}\right)^{2}\right]^{-1}\right\}+\frac{\partial R}{\partial p_{i}}, \\
\dot{p}_{i}= & \bar{F}_{i}\left(t, q^{i}, p_{i},\{\sigma\}_{\mu}\right)-g \sum_{j=i}^{76-i} \bar{L}_{j} \bar{m}_{j} \sin q^{j} \\
& -\sum_{j=i}^{76-i} \bar{L}_{j} \sin q^{j} p_{i} p_{j}\left[\bar{m}_{i}\left(\sum_{k=1}^{i} \bar{L}_{k} \cos q^{k}\right)^{3}\right]^{-1}+\frac{\partial R}{\partial q^{i}}, \\
q^{i}(0)= & \bar{q}_{0}^{i}, \quad p_{i}(0)=\bar{p}_{i}^{0} \quad(i=1, \ldots, 75) .
\end{aligned}
$$

Applying stochastic forces, including diffusion fluctuations $B_{i j}\left[q^{i}(t), t\right]$ and discontinuous jumps in the form of $N$-dimensional Wiener process $W^{j}(t)$, to $\mu$-Hamiltonian $\mathrm{HB}$ system (3.9), we obtain fuzzy-stochastic $[\mu \sigma]$-Hamiltonian $\operatorname{HB}$ system $[15,19]$

$$
\begin{gathered}
d q^{i}=\left(\frac{\partial H_{0}\left(q, p, \sigma_{\mu}\right)}{\partial p_{i}}+\frac{\partial R}{\partial p_{i}}\right) d t, \\
d p_{i}=B_{i j}\left[q^{i}(t), t\right] d W^{j}(t) \\
+\left(\bar{F}_{i}-\frac{\partial H_{0}\left(q, p, \sigma_{\mu}\right)}{\partial q^{i}}+\frac{\partial R}{\partial q^{i}}\right) d t, \\
q^{i}(0)=\bar{q}_{0}^{i}, \quad p_{i}(0)=\bar{p}_{i}^{0} .
\end{gathered}
$$


Particularly, in the spinal HB simulator, the velocity and force $[\mu \sigma]$-Hamiltonian $\mathrm{HB}$ equations (3.11), (3.12), and (3.13) become

$$
\begin{gathered}
d q^{i}=\left(p_{i}\left\{\left[\bar{J}_{i}\right]^{-1}+\left[\bar{m}_{i}\left(\sum_{j=1}^{i} \bar{L}_{j} \cos q^{j}\right)^{2}\right]^{-1}\right\}+\frac{\partial R}{\partial p_{i}}\right) d t \\
d p_{i}=B_{i j}\left[q^{i}(t), t\right] d W^{j}(t)+\left(\bar{F}_{i}\left(t, q^{i}, p_{i},\{\sigma\}_{\mu}\right)-g \sum_{j=i}^{76-i} \bar{L}_{j} \bar{m}_{j} \sin q^{j}\right. \\
\left.-\sum_{j=i}^{76-i} \bar{L}_{j} \sin q^{j} p_{i} p_{j}\left[\bar{m}_{i}\left(\sum_{k=1}^{i} \bar{L}_{k} \cos q^{k}\right)^{3}\right]^{-1}+\frac{\partial R}{\partial q^{i}}\right) d t \\
q^{i}(0)=\bar{q}_{0}^{i}, \quad p_{i}(0)=\bar{p}_{i}^{0} \quad(i=1, \ldots, 75) .
\end{gathered}
$$

\section{Spinal control level}

Our first task is to establish the nonlinear loop functor $L=\Xi[\mathscr{L}]: \mathscr{E} \mathscr{X} \Rightarrow \mathscr{E} \mathscr{X}^{*}$ on the category $\mathscr{E} \mathscr{X}$ of the spinal FC level.

Control Hamiltonian function $H_{c}: T^{*} M^{N} \rightarrow \mathbb{R}$ of FC is in local canonical coordinates on $T^{*} M^{N}$ defined by [24]

$$
H_{c}(q, p, u)=H_{0}(q, p)-q^{i} u_{i} \quad(i=1, \ldots, N),
$$

where $u_{i}=u_{i}(t, q, p)$ are feedback-control one-forms representing the spinal FC level $u$ corrections to the covariant torques $F_{i}=F_{i}(t, q, p)$.

Using $\delta$-Hamiltonian HB system (3.1), (3.2), and (3.3) and the control Hamiltonian function (4.1), the $c_{\delta}$-Hamiltonian FC system can be defined as

$$
\begin{gathered}
\dot{q}^{i}=\frac{\partial H_{c}(q, p, u)}{\partial p_{i}}+\frac{\partial R(q, p)}{\partial p_{i}}, \\
\dot{p}_{i}=F_{i}-\frac{\partial H_{c}(q, p, u)}{\partial q^{i}}+\frac{\partial R(q, p)}{\partial q^{i}}, \\
o^{i}=-\frac{\partial H_{c}(q, p, u)}{\partial u_{i}}, \\
q^{i}(0)=q_{0}^{i}, \quad p_{i}(0)=p_{i}^{0} \quad(i=1, \ldots, N),
\end{gathered}
$$

where $o^{i}=o^{i}(t)$ represent FC natural outputs which can be different from commonly used joint angles.

If nominal reference outputs $o_{R}^{i}=o_{R}^{i}(t)$ are known, the simple PD stiffness servo [32] could be formulated, via error function $e(t)=o^{j}-o_{R}^{j}$, in covariant form

$$
u_{i}=K_{o} \delta_{i j}\left(o^{j}-o_{R}^{j}\right)+K_{\dot{o}} \delta_{i j}\left(\dot{o}^{j}-\dot{o}_{R}^{j}\right)
$$

where $K$ s are the control gains and $\delta_{i j}$ is the Kronecker tensor. 
If natural outputs $o^{i}$ actually are the joint angles and nominal canonical trajectories $\left(q_{R}^{i}=q_{R}^{i}(t), p_{i}^{R}=p_{i}^{R}(t)\right)$ are known, then the stiffness servo (4.3) could be formulated in canonical form as

$$
u_{i}=K_{q} \delta_{i j}\left(q^{i}-q_{R}^{i}\right)+K_{p}\left(p_{i}-p_{i}^{R}\right)
$$

Using the fuzzified $\mu$-Hamiltonian HB system (3.9) and the control Hamiltonian function (4.1), the fuzzified $c_{\mu}$-Hamiltonian FC system can be defined as

$$
\begin{gathered}
\dot{q}^{i}=\frac{\partial H_{c}\left(q, p, u, \sigma_{\mu}\right)}{\partial p_{i}}+\frac{\partial R(q, p)}{\partial p_{i}}, \\
\dot{p}_{i}=\bar{F}_{i}-\frac{\partial H_{c}\left(q, p, u, \sigma_{\mu}\right)}{\partial q^{i}}+\frac{\partial R(q, p)}{\partial q^{i}}, \\
\bar{o}^{i}=-\frac{\partial H_{c}\left(q, p, u, \sigma_{\mu}\right)}{\partial u_{i}}, \\
q^{i}(0)=\bar{q}_{0}^{i}, \quad p_{i}(0)=\bar{p}_{i}^{0} \quad(i=1, \ldots, N),
\end{gathered}
$$

where $\bar{o}^{i}=\bar{o}^{i}(t)$ represent the fuzzified natural outputs.

Using the fuzzy-stochastic $[\mu \sigma]$-Hamiltonian HB system (3.9) and (3.13) and the control Hamiltonian function (4.1), the fuzzy-stochastic $c_{\mu \sigma}$-Hamiltonian FC system can be defined as

$$
\begin{gathered}
d q^{i}=\left(\frac{\partial H_{c}\left(q, p, u, \sigma_{\mu}\right)}{\partial p_{i}}+\frac{\partial R(q, p)}{\partial p_{i}}\right) d t \\
d p_{i}=B_{i j}\left[q^{i}(t), t\right] d W^{j}(t) \\
+\left(\bar{F}_{i}-\frac{\partial H_{c}\left(q, p, u, \sigma_{\mu}\right)}{\partial q^{i}}+\frac{\partial R(q, p)}{\partial q^{i}}\right) d t \\
d \bar{o}^{i}=-\frac{\partial H_{c}\left(q, p, u, \sigma_{\mu}\right)}{\partial u_{i}} d t \\
q^{i}(0)=\bar{q}_{0}^{i}, \quad p_{i}(0)=\bar{p}_{i}^{0} \quad(i=1, \ldots, N) .
\end{gathered}
$$

If we have the case that not all of the configuration joints on the configuration manifold $M^{N}$ are active in the specified robot task, we can introduce the coupling Hamiltonians $H^{j}=H^{j}(q, p), j=1, \ldots, M \leq N$, corresponding to the system's active joints, and we come to the affine Hamiltonian function $H_{a}: T^{*} M^{N} \rightarrow \mathbb{R}$, in local canonical coordinates on $T^{*} M^{N}$ given as [24]

$$
H_{a}(q, p, u)=H_{0}(q, p)-H^{j}(q, p) u_{j}
$$


Again, using $\delta$-Hamiltonian HB system (3.1), (3.2), and (3.3) and the affine Hamiltonian function (4.7), affine $a_{\delta}$-Hamiltonian FC system can be defined as

$$
\begin{gathered}
\dot{q}^{i}=\frac{\partial H_{0}(q, p)}{\partial p_{i}}-\frac{\partial H^{j}(q, p)}{\partial p_{i}} u_{j}+\frac{\partial R}{\partial p_{i}}, \\
\dot{p}_{i}=F_{i}-\frac{\partial H_{0}(q, p)}{\partial q^{i}}+\frac{\partial H^{j}(q, p)}{\partial q^{i}} u_{j}+\frac{\partial R}{\partial q^{i}}, \\
o^{i}=-\frac{\partial H_{a}(q, p, u)}{\partial u_{i}}=H^{j}(q, p), \\
q^{i}(0)=q_{0}^{i}, \quad p_{i}(0)=p_{i}^{0} \quad(i=1, \ldots, N ; j=1, \ldots, M \leq N) .
\end{gathered}
$$

Let $F(M)$ denote the set of all smooth (i.e., $C^{\infty}$ ) real-valued functions $f: M \rightarrow \mathbb{R}$ on a smooth manifold $M, V(M)$, the set of all smooth vector fields on $M$, and $V^{*}(M)$, the set of all differential one-forms on $M$. Also, let the vector field $\zeta \in V(M)$ be given with its local flow $\phi_{t}: M \rightarrow M$ such that at a point $x \in M, d /\left.d t\right|_{t=0} \phi_{t} x=\zeta(x)$, and $\phi_{t}^{*}$ represents the pullback by $\phi_{t}$. Recall that the Lie derivative differential operator $L_{\zeta}$ is defined

(i) on a function $f \in F(M)$ as $L_{\zeta}: F(M) \rightarrow F(M), L_{\zeta} f=\left.(d / d t)\left(\phi_{t}^{*} f\right)\right|_{t=0}$,

(ii) on a vector field $\eta \in V(M)$ as $L_{\zeta}: V(M) \rightarrow V(M), L_{\zeta} \eta=\left.(d / d t)\left(\phi_{t}^{*} \eta\right)\right|_{t=0} \equiv[\zeta, \eta]$ -the Lie bracket,

(iii) on a one-form $\alpha \in V^{*}(M)$ as $L_{\zeta}: V^{*}(M) \rightarrow V^{*}(M), L_{\zeta} \alpha=\left.(d / d t)\left(\phi_{t}^{*} \alpha\right)\right|_{t=0}$.

In general, for any smooth tensor field $\mathbf{T}$ on $M$, the Lie derivative $L_{\zeta} \mathbf{T}$ geometrically represents a directional derivative of $\mathbf{T}$ along the flow $\phi_{t}$. Using the Lie derivative formalism, and applying the constant relative degree $r$ (see $[11,24]$ ) to all $N$ joints of the affine $a_{\delta}$-Hamiltonian FC system (4.8), the control law for asymptotic tracking the reference outputs $o_{R}^{j}$ could be formulated as (generalized from [11])

$$
u_{j}=\frac{\dot{o}_{R}^{(r) j}-L_{f}^{(r)} H^{j}+\sum_{s=1}^{r} c_{s-1}\left(o_{R}^{(s-1) j}-L_{f}^{(s-1)} H^{j}\right)}{L_{g} L_{f}^{(r-1)} H^{j}},
$$

where standard MIMO vector fields $f$ and $g$ are given by

$$
f=\left(\frac{\partial H_{0}}{\partial p_{i}},-\frac{\partial H_{0}}{\partial q^{i}}\right), \quad g=\left(-\frac{\partial H^{j}}{\partial p_{i}}, \frac{\partial H^{j}}{\partial q^{i}}\right)
$$

and $c_{s-1}$ are the coefficients of linear differential equation of order $r$ for the error function $e(t)=o^{j}-o_{R}^{j}$,

$$
e^{(r)}+c_{r-1} e^{(r-1)}+\cdots+c_{1} e^{(1)}+c_{0} e=0
$$


Using the fuzzified $\mu$-Hamiltonian HB system (3.9) and the affine Hamiltonian function (4.7), the fuzzy affine $a_{\mu}$-Hamiltonian FC system can be defined as

$$
\begin{gathered}
\dot{q}^{i}=\frac{\partial H_{0}\left(q, p, \sigma_{\mu}\right)}{\partial p_{i}}-\frac{\partial H^{j}\left(q, p, \sigma_{\mu}\right)}{\partial p_{i}} u_{j}+\frac{\partial R(q, p)}{\partial p_{i}}, \\
\dot{p}_{i}=\bar{F}_{i}-\frac{\partial H_{0}\left(q, p, \sigma_{\mu}\right)}{\partial q^{i}}+\frac{\partial H^{j}\left(q, p, \sigma_{\mu}\right)}{\partial q^{i}} u_{j}+\frac{\partial R(q, p)}{\partial q^{i}}, \\
\bar{o}^{i}=-\frac{\partial H_{a}\left(q, p, u, \sigma_{\mu}\right)}{\partial u_{i}}=H^{j}\left(q, p, \sigma_{\mu}\right), \\
q^{i}(0)=\bar{q}_{0}^{i}, \quad p_{i}(0)=\bar{p}_{i}^{0} \quad(i=1, \ldots, N ; j=1, \ldots, M \leq N) .
\end{gathered}
$$

Finally, using the fuzzy-stochastic $[\mu \sigma]$-Hamiltonian HB system (3.11), (3.12), and (3.13) and the affine Hamiltonian function (4.7), we obtain the fuzzy-stochastic affine $a_{\mu \sigma}$-Hamiltonian FC system defined as

$$
\begin{array}{r}
d q^{i}=\left(\frac{\partial H_{0}\left(q, p, \sigma_{\mu}\right)}{\partial p_{i}}-\frac{\partial H^{j}\left(q, p, \sigma_{\mu}\right)}{\partial p_{i}} u_{j}+\frac{\partial R(q, p)}{\partial p_{i}}\right) d t \\
d p_{i}=B_{i j}\left[q^{i}(t), t\right] d W^{j}(t) \\
+\left(\bar{F}_{i}-\frac{\partial H_{0}\left(q, p, \sigma_{\mu}\right)}{\partial q^{i}}+\frac{\partial H^{j}\left(q, p, \sigma_{\mu}\right)}{\partial q^{i}} u_{j}+\frac{\partial R(q, p)}{\partial q^{i}}\right) d t \\
d \bar{o}^{i}=-\frac{\partial H_{a}\left(q, p, u, \sigma_{\mu}\right)}{\partial u_{i}} d t=H^{j}\left(q, p, \sigma_{\mu}\right) d t \\
q^{i}(0)=\bar{q}_{0}^{i}, \quad p_{i}(0)=\bar{p}_{i}^{0} \quad(i=1, \ldots, N ; j=1, \ldots, M \leq N) .
\end{array}
$$

Being of high degree and highly nonlinear, all of these affine control systems are extremely sensitive upon the variation of parameters, inputs, and initial conditions. The sensitivity function $S$ of the affine Hamiltonian $H_{a}(q, p, u)$ to the parameters $\beta_{i}$ (including the segment lengths $L_{i}$, masses $m_{i}$, moments of inertia $J_{i}$, and joint dampings $b_{i}$, see $[15,17,18,19])$ is in the case of $a_{\delta}$-Hamiltonian FC system defined as

$$
S(H, \beta)=\frac{\beta_{i}}{H_{a}(q, p, u)} \frac{\partial H_{a}(q, p, u)}{\partial \beta_{i}}
$$

and similarly in other two $a_{\mu}$ and $a_{\mu \sigma}$ cases.

The three affine FC level systems $a_{\delta}, a_{\mu}$, and $a_{\mu \sigma}$ resemble (in a fuzzy-stochasticHamiltonian form) Houk's autogenetic motor servo of muscle spindle and Golgi tendon [9], correcting the covariant driving torques $F_{i}=F_{i}(t, q, p)$ by local "reflex controls" $u_{i}(t, q, p)$. They form the nonlinear loop functor $L=\Xi[\mathscr{L}]: \mathscr{E} \mathscr{X} \Rightarrow \mathscr{E} \mathscr{X}^{*}$. For its computer-algebra implementation, see the appendix. 


\section{Cerebellar control level}

Our second task is to establish the nonlinear loop functor $L=\Xi[\mathscr{L}]: \mathscr{T} \mathscr{A} \Rightarrow \mathscr{T} \mathscr{A}^{*}$ on the category $\mathscr{T} \mathscr{A}$ of the cerebellar FC level. Here we propose an oscillatory neurodynamical $(x, y, \boldsymbol{\omega})$-system (adapted from [13]), a bidirectional, self-organized, associative-memory machine, resembling the function of a set of excitatory granule cells and inhibitory Purkinje cells in the middle layer of the cerebellum [10]. The neurodynamical $(x, y, \boldsymbol{\omega})$-system acts on neural-image manifold $M_{i m}^{N}$ of the configuration manifold $M^{N}$ as a pair of smooth, "1 - 1" and "onto" maps $\left(\Psi, \Psi^{-1}\right)$, where $\Psi: M^{N} \rightarrow M_{i m}^{N}$ represents the feedforward map, and $\Psi^{-1}: M_{i m}^{N} \rightarrow M^{N}$ represents the feedback map. Locally, it is defined in Riemannian neural coordinates $x^{i}, y_{i} \in V_{y}$ on $M_{i m}^{N}$, which are in bijective correspondence with symplectic joint coordinates $q^{i}, p_{i} \in U_{p}$ on $T^{*} M^{N}$.

The $(x, y, \boldsymbol{\omega})$-system is formed out of two distinct, yet nonlinearly coupled neural subsystems, with $A^{i}(q)(5.1)$ and $B_{i}(p)(5.2)$ as system inputs, and the feedback-control oneforms $u_{i}(5.9)$ as system outputs:

(i) granule cells excitatory (contravariant) and Purkinje cells inhibitory (covariant) activation $(x, y)$-dynamics (5.1), (5.2), (5.3), and (5.4), defined, respectively, by a vector field $x^{i}=x^{i}(t): M_{i m}^{N} \rightarrow T M_{i m}^{N}$ representing a cross-section of the tangent bundle $T M_{i m}^{N}$, and a one-form $y_{i}=y_{i}(t): M_{i m}^{N} \rightarrow T^{*} M_{i m}^{N}$ representing a crosssection of the cotangent bundle $T^{*} M_{i m}^{N}$;

(ii) excitatory and inhibitory unsupervised learning ( $\boldsymbol{\omega})$-dynamics (5.5) and (5.6) generated by random differential Hebbian learning process (5.7) and (5.8), defined, respectively, by contravariant synaptic tensor field $\omega^{i j}=\omega^{i j}(t): M_{i m}^{N} \rightarrow$ $T T M_{i m}^{N}$ and covariant synaptic tensor field $\omega_{i j}=\omega_{i j}(t): M_{i m}^{N} \rightarrow T^{*} T^{*} M_{i m}^{N}$ representing cross-sections of contravariant and covariant tensor bundles, respectively.

The system of $N$ equations ( $N$ being the same as the number of mechanical degrees of freedom) is

$$
\begin{aligned}
\dot{x}^{i} & =A^{i}(q)+\omega^{i j} f_{j}(y)-x^{i}, \\
\dot{y}_{i} & =B_{i}(p)+\omega_{i j} f^{j}(x)-y_{i}, \\
A^{i}(q) & =K_{q}\left(q^{i}-q_{R}^{i}\right), \\
B_{i}(p) & =K_{p}\left(p_{i}^{R}-p_{i}\right), \\
\dot{\omega}^{i j} & =-\omega^{i j}+I^{i j}(x, y), \\
\dot{\omega}_{i j} & =-\omega_{i j}+I_{i j}(x, y), \\
I^{i j} & =f^{i}(x) f^{j}(y)+\dot{f}^{i}(x) \dot{f}^{j}(y)+\sigma^{i j}, \\
I_{i j} & =f_{i}(x) f_{j}(y)+\dot{f}_{i}(x) \dot{f}_{j}(y)+\sigma_{i j}, \\
u_{i} & =\frac{1}{2}\left(\delta_{i j} x^{i}+y_{i}\right) \quad(i, j=1, \ldots, N) .
\end{aligned}
$$

Here $\boldsymbol{\omega}$ is a symmetric second-order synaptic tensor field; $I^{i j}=I^{i j}(x, y, \sigma)$ and $I_{i j}=$ $I_{i j}(x, y, \sigma)$, respectively, denote contravariant-excitatory and covariant-inhibitory random 
differential Hebbian innovation functions with tensorial Gaussian noise $\boldsymbol{\sigma}$ (in both variances); $f$ s and $\dot{f}$ s denote sigmoid activation functions $(f=\tanh (\cdot))$ and corresponding signal velocities $\left(\dot{f}=1-f^{2}\right)$, respectively, in both variances.

$A^{i}(q)$ and $B_{i}(p)$ are contravariant-excitatory and covariant-inhibitory neural inputs to granule and Purkinje cells, respectively; $u_{i}$ are the corrections to the feedback-control one-forms on the cerebellar FC level.

Nonlinear activation $(x, y)$-dynamics (5.1), (5.2), (5.3), and (5.4), describes a twophase biological neural oscillator field, in which the excitatory neural field excites the inhibitory neural field, which itself reciprocally inhibits the excitatory one. $(x, y)$-dynamics represents a nonlinear extension of a linear, Lyapunov stable, conservative, gradient system defined in local neural coordinates $x^{i}, y_{i} \in V_{y}$ on $T^{*} M_{i m}^{N}$ as

$$
\dot{x}^{i}=-\frac{\partial \Phi}{\partial y_{i}}=\omega^{i j} y_{j}-x^{i}, \quad \dot{y}_{i}=-\frac{\partial \Phi}{\partial x^{i}}=\omega_{i j} x^{j}-y_{i} .
$$

The gradient system (5.10) is derived from scalar, neuro-synaptic action potential $\Phi$ : $T^{*} M^{N} \rightarrow \mathbb{R}$, given by a negative, smooth bilinear form in $x^{i}, y_{i} \in V_{y}$ on $T^{*} M_{i m}^{N}$ as

$$
-2 \Phi=\omega_{i j} x^{i} x^{j}+\omega^{i j} y_{i} y_{j}-2 x^{i} y_{i} \quad(i, j=1, \ldots, N),
$$

which itself represents a $\Psi$-image of the Riemannian metrics $g: T M^{N} \rightarrow \mathbb{R}$ on the configuration manifold $M^{N}$.

The nonlinear oscillatory activation ( $x, y)$-dynamics (5.1), (5.2), (5.3), and (5.4) is obtained from the linear conservative dynamics (5.10) by adding configuration-dependent inputs $A^{i}$ and $B_{i}$, as well as sigmoid activation functions $f_{j}$ and $f^{j}$, respectively. It represents an interconnected pair of excitatory and inhibitory neural fields.

Both variant forms of learning $(\boldsymbol{\omega})$-dynamics (5.5) and (5.6) are given by a generalized unsupervised (self-organizing) Hebbian learning scheme (see [21]), in which $\dot{\omega}_{i j}$ (resp., $\dot{\omega}^{i j}$ ) denotes the new update value, $-\omega_{i j}$ (resp., $\omega^{i j}$ ) corresponds to the old value and $I_{i j}\left(x^{i}, y_{j}\right)$ (resp., $\left.I^{i j}\left(x^{i}, y_{j}\right)\right)$ is the innovation function of the symmetric second-order synaptic tensor field $\boldsymbol{\omega}$. The nonlinear innovation functions $I_{i j}$ and $I^{i j}$ are defined by random differential Hebbian learning process (5.7) and (5.8). As $\boldsymbol{\omega}$ is a symmetric and zero-trace coupling synaptic tensor, the conservative linear activation dynamics (5.10) is equivalent to the rule that "the state of each neuron (in both neural fields) is changed in time if and only if the scalar action potential $\Phi(5.11)$, is lowered." Therefore, the scalar action potential $\Phi$ represents the monotonically decreasing Lyapunov function (such that $\dot{\Phi} \leq 0$ ) for the conservative linear dynamics (5.10), which converges to a local minimum or ground state of $\Phi$. That is to say, the system (5.10) moves in the direction of decreasing the scalar action potential $\Phi$, and when both $\dot{x}^{i}=0$ and $\dot{y}_{i}=0$ for all $i=1, \ldots, N$, the steady state is reached.

In this way, the neurodynamical $(x, y, \boldsymbol{\omega})$-system acts as tensor-invariant selforganizing (excitatory/inhibitory) associative memory machine, resembling the set of 
granule and Purkinje cells of cerebellum [10], and generalizing Kosko's BAM (as well as ABAM and RABAM) model

$$
\begin{aligned}
& \dot{v}_{j}=-a_{j}\left(v_{j}\right)\left[b_{j}\left(v_{j}\right)-\sum_{k=1}^{N} f_{k}\left(v_{k}\right) m_{j k}\right], \\
& \dot{u}_{k}=-a_{k}\left(u_{k}\right)\left[b_{k}\left(u_{k}\right)-\sum_{j=1}^{N} f_{j}\left(u_{j}\right) m_{j k}\right],
\end{aligned}
$$

(for $j, k=1, \ldots, N$ ), which is globally stable for the cases of signal and random-signal Hebbian learning (see [21]).

The feedforward map $\Psi: M^{N} \rightarrow M_{i m}^{N}$ is realized by the inputs $A^{i}(q)$ and $B_{i}(p)$ to the $(x, y, \boldsymbol{\omega})$-system, while the feedback map $\Psi^{-1}: M_{i m}^{N} \rightarrow M^{N}$ is realized by the system output, that is, the feedback-control one-forms $u_{i}(x, y)$. These represent the cerebellar FC level corrections to the covariant torques $F_{i}=F_{i}(t, q, p)$.

The tensor-invariant form of the oscillatory neurodynamical $(x, y, \omega)$-system $(5.5)$ and (5.6) resembles the associative action of the granule and Purkinje cells in the tunning of the limb cortico-rubro-cerebellar recurrent network [10], giving the cerebellar correction $u_{i}(x, y)$ to the covariant driving torques $F_{i}=F_{i}(t, q, p)$. In this way, the $(x, y, \omega)$-system forms the nonlinear loop functor $L=\Xi[\mathscr{L}]: \mathscr{T} \mathscr{A} \Rightarrow \mathscr{T} \mathscr{A}^{*}$. For its computer-algebra implementation, see the appendix.

\section{Cortical control level}

Our third task is to establish the nonlinear loop functor $L=\Xi[\mathscr{L}]: \mathscr{Y} \mathcal{T} \Rightarrow \mathscr{S} \mathcal{T}^{*}$ on the category $\mathscr{S} \mathcal{T}$ of the cortical FC level.

For the purpose of our cortical control, the humanoid configuration manifold $M^{N}$, depicted in Figure 3.1, could be first reduced to $\mathrm{N}$-torus, and second transformed to $\mathrm{N}$ cube ("hyper-joystick"), using the following topological techniques (see $[14,15,18,19]$ ).

Let $S^{1}$ denote the constrained unit circle in the complex plane, which is an Abelian Lie group. Firstly, we propose two reduction homeomorphisms, using the semidirect product $\ltimes$ of the constrained $\mathrm{SO}(2)$-groups:

$$
\mathrm{SO}(3) \approx \mathrm{SO}(2) \ltimes \mathrm{SO}(2) \ltimes \mathrm{SO}(2), \quad \mathrm{SO}(2) \approx S^{1} .
$$

Next, let $I^{N}$ be the unit cube $[0,1]^{N}$ in $\mathbb{R}^{N}$ and “ " an equivalence relation on $\mathbb{R}^{N}$ obtained by "gluing" together the opposite sides of $I^{N}$, preserving their orientation. Therefore, $M^{N}$ can be represented as the quotient space of $\mathbb{R}^{N}$ by the space of the integral lattice points in $\mathbb{R}^{N}$, that is, an oriented and constrained $N$-dimensional torus $T^{N}$ :

$$
\mathbb{R}^{N} / Z^{N}=I^{N} / \sim \approx \prod_{i=1}^{N} S_{i}^{1} \equiv\left\{\left(q^{i}, i=1, \ldots, N\right): \bmod 2 \pi\right\}=T^{N}
$$


Its Euler-Poincaré characteristic is (by the de Rham theorem), both for $T^{N}$ and its momentum phase space $T^{*} T^{N}$, given by (see [15])

$$
\chi\left(T^{N}, T^{*} T^{N}\right)=\sum_{p=1}^{N}(-1)^{p} b_{p},
$$

where $b_{p}$ are the Betti numbers defined as

$$
\begin{gathered}
b^{0}=1, \\
b^{1}=N, \ldots, b^{p}=\left(\begin{array}{c}
N \\
p
\end{array}\right), \ldots, b^{N-1}=N, \\
b^{N}=1 \quad(0 \leq p \leq N) .
\end{gathered}
$$

In our example case of the spinal HB simulator (depicted in Figure 3.2), the configuration torus $T^{N}$, defined by (6.2), becomes $T^{75}$. In the same way, the momentum phasespace manifold $T^{*} M$ can be represented by $T^{*} T^{N}$, which in case of the spinal HB simulator becomes $T^{*} T^{75}$.

Conversely, by "ungluing" the configuration space, we obtain the primary unit cube. Let “ * " denote an equivalent decomposition or "ungluing" relation. By the Tychonoff product-topology theorem, for every such quotient space, there exists a "selector" such that its quotient models are homeomorphic, that is, $T^{N} / \sim^{*} \approx A^{N} / \sim^{*}$. Therefore, $I_{q}^{N}$ represents a "selector" for the configuration torus $T^{N}$ and can be used as an $N$-directional " $\hat{q}$-command-space" for FC. Any subset of degrees of freedom on the configuration torus $T^{N}$ representing the joints included in HB has its simple, rectangular image in the rectified $\hat{q}$-command space selector $I_{q}^{N}$, and any joint angle $q^{i}$ has its rectified image $\hat{q}^{i}$.

In the case of an end-effector, $\hat{q}^{i}$ reduces to the position vector in external Cartesian coordinates $z^{r}(r=1, \ldots, 3)$. If orientation of the end-effector can be neglected, this gives a topological solution to the standard inverse kinematics problem.

Analogously, all momenta $\hat{p}_{i}$ have their images as rectified momenta $\hat{p}_{i}$ in the $\hat{p}$ command space selector $I_{p}^{N}$. Therefore, the total momentum phase-space manifold $T^{*} T^{N}$ obtains its "cortical image" as the $\widehat{(q, p)}$-command space, a trivial $2 N$-dimensional bundle $I_{q}^{N} \times I_{p}^{N}$.

Now, the simplest way to perform the feedback FC on the cortical $\widehat{(q, p)}$-command space $I_{q}^{N} \times I_{p}^{N}$, and also to mimic the cortical-like behavior (see $\left.[2,4,25,26]\right)$ is to use the $2 \mathrm{~N}$-dimensional fuzzy-logic controller, in pretty much the same way as in the popular “inverted pendulum” examples (see [21]).

We propose the fuzzy feedback-control map $\Xi$ that maps all the rectified joint angles and momenta into the feedback-control one-forms

$$
\Xi:\left(\hat{q}^{i}(t), \hat{p}_{i}(t)\right) \longmapsto u_{i}(t, q, p)
$$


so that their corresponding universes of discourse, $\hat{Q}^{i}=\left(\hat{q}_{\max }^{i}-\hat{q}_{\min }^{i}\right), \hat{P}_{i}=\left(\hat{p}_{i}^{\max }-\hat{p}_{i}^{\min }\right)$ and ${ }_{i}=\left(u_{i}^{\max }-u_{i}^{\min }\right)$, respectively, are mapped as

$$
\Xi: \prod_{i=1}^{N} \hat{Q}^{i} \times \prod_{i=1}^{N} \hat{P}_{i} \longrightarrow \prod_{i=1}^{N}
$$

The $2 N$-dimensional map $\Xi(6.5)$ and (6.6) represents a fuzzy inference system, defined by (adapted from [12]) the following.

(1) Fuzzification of the crisp rectified and discretized angles, momenta and controls using Gaussian-bell membership functions

$$
\mu_{k}(\chi)=\exp \left[-\frac{\left(\chi-m_{k}\right)^{2}}{2 \sigma_{k}}\right] \quad(k=1,2, \ldots, 9),
$$

where $\chi \in D$ is the common symbol for $\hat{q}^{i}, \hat{p}_{i}$, and $u_{i}(q, p)$ and $D$ is the common symbol for $\hat{Q}^{i}, \hat{P}_{i}$ and $i$; the mean values $m_{k}$ of the nine partitions of each universe of discourse $D$ are defined as $m_{k}=\lambda_{k} D+\chi_{\min }$, with partition coefficients $\lambda_{k}$ uniformly spanning the range of $D$, corresponding to the set of nine linguistic variables $L=\{N L, N B, N M, N S, Z E, P S, P M, P B, P L\}$; standard deviations are kept constant $\sigma_{k}=D / 9$. Using the linguistic vector $L$, the $9 \times 9$ FAM (fuzzy associative memory) matrix (a "linguistic phase plane") is heuristically defined for each humanoid joint in a symmetrical weighted form

$$
\mu_{k l}=\omega_{k l} \exp \left\{-50\left[\lambda_{k}+u(q, p)\right]^{2}\right\}, \quad(k, l=1, \ldots, 9)
$$

with weights $\varpi_{k l} \in\{0.6,0.6,0.7,0.7,0.8,0.8,0.9,0.9,1.0\}$.

(2) Mamdani inference is used on each FAM matrix $\mu_{k l}$ for all humanoid joints.

(i) $\mu\left(\hat{q}^{i}\right)$ and $\mu\left(\hat{p}_{i}\right)$ are combined inside the fuzzy IF-THEN rules using AND (intersection or minimum) operator,

$$
\mu_{k}\left[\bar{u}_{i}(q, p)\right]=\min _{l}\left\{\mu_{k l}\left(\hat{q}^{i}\right), \mu_{k l}\left(\hat{p}_{i}\right)\right\} .
$$

(ii) The output sets from different IF-THEN rules are then combined using OR (union or maximum) operator to get the final output, fuzzy-covariant torques,

$$
\mu\left[u_{i}(q, p)\right]=\max _{k}\left\{\mu_{k}\left[\bar{u}_{i}(q, p)\right]\right\} .
$$

(3) Defuzzification of the fuzzy controls $\mu\left[u_{i}(q, p)\right]$ with the "center-of-gravity" method

$$
u_{i}(q, p)=\frac{\int \mu\left[u_{i}(q, p)\right] d u_{i}}{\int d u_{i}}
$$

to update the crisp feedback-control one-forms $u_{i}=u_{i}(t, q, p)$. These represent the cortical FC level corrections to the covariant torques $F_{i}=F_{i}(t, q, p)$. 
Operationally, the construction of the cortical $\widehat{(q, p)}$-command space $I_{q}^{N} \times I_{p}^{N}$ and the $2 N$-dimensional feedback map $\Xi(6.5)$ and (6.6) mimic the regulation of locomotor conditioned reflexes by the motor cortex [10], giving the cortical correction to the covariant driving torques $F_{i}$. Together, they form the nonlinear loop functor $L=\Xi[\mathscr{L}]: \mathscr{Y T} \Rightarrow$ $\mathscr{S} \mathscr{T}^{*}$. For its computer-algebra implementation, see the appendix.

\section{Translational control of IK chains}

Besides providing the subtle rotational $u_{i}=u_{i}(t, q, p)$-corrections for the covariant joint torques $F_{i}=F_{i}(t, q, p)$, both the cerebellar and the cortical control systems can be extended to provide translational control for moving the whole 6-DOF IK chains (like a humanoid arm or leg) (see [4]).

For the sake of translational control of IK chains, both input functions $A^{i}(q)$ and $B_{i}(p)$ in the cerebellar FC system defined by 5.3 and (5.4) have to be extended to $A^{i}\left[\sum_{k=1}^{6} q_{k}\right]$ and $B_{i}\left[\sum_{k=1}^{6} p^{k}\right]$. The output feedback-control one-forms $u_{i}^{\mathrm{IK}}$ will then represent corrections for the resulting translational forces $F_{i}^{\mathrm{IK}}$ moving the whole IK chains.

Similarly, for the sake of the cortical translational control of IK chains, (6.5) and (6.6) have to be extended to

$$
\Xi:\left(\sum_{k=1}^{6} \hat{q}^{i}(t), \sum_{k=1}^{6} \hat{p}_{i}(t)\right) \longmapsto u_{i}\left(t, \sum_{k=1}^{6} q, \sum_{k=1}^{6} p\right)
$$

so that their corresponding universes of discourse are mapped as

$$
\Xi: \prod_{i=1}^{N} \sum_{k=1}^{6} \hat{Q}^{i} \times \prod_{i=1}^{N} \sum_{k=1}^{6} \hat{P}_{i} \longrightarrow \prod_{i=1}^{N}{ }_{i}^{\mathrm{IK}}
$$

\section{Conclusion}

This paper proposes the new, brain-like, hierarchical (affine-neuro-fuzzy-topological) control for the previously developed biomechanically realistic humanoid robot dynamics. The categorical form of a tensor-invariant, "meta-cybernetic" functor machine has been used to develop the brain-like controller. It comprises the three-level, nonlinear feedback control of muscle-like actuators, as implemented in the full-spine simulator, developed by the authors (see [35]). On the spinal level, nominal joint-trajectory tracking is formulated as an affine Hamiltonian control system, resembling the spinal (autogenetic-reflex) "motor servo." On the cerebellar level, a feedback-control map is proposed in the form of self-organized, oscillatory, neurodynamical system, resembling the associative interaction of excitatory granule cells and inhibitory Purkinje cells. On the cortical level, a topological "hyper-joystick" command space, is formulated with a fuzzy-logic feedback-control map defined on it, resembling the regulation of locomotor conditioned reflexes. Finally, both the cerebellar and the cortical control systems are extended to provide the translational force control for moving 6-degree-of-freedom chains of inverse kinematics. The computer-algebra implementation is given on reader's request. 


\section{References}

[1] O. A. Bauchau and L. Trainelli, The vectorial parameterization of rotation, Nonlinear Dynam. 32 (2003), no. 1, 71-92.

[2] A. Billard and M. Mataric, Learning human arm movements by imitation: Evaluation of a biologically-inspired connectionist architecture, Rob. Auton. Syst. 941 (2001), 1-16.

[3] F. Crepel, N. Hemart, D. Jaillard, and H. Daniel, Long-term depression in the cerebellum, The Handbook of Brain Theory and Neural Networks (M. Arbib, ed.), MIT Press, Massachusetts, 2nd ed., 1998, pp. 560-563.

[4] A. D'Souza, S. Vijayakumar, and S. Schaal, Learning inverse kinematics, Proc. IEEE/RSJ International Conference on Intelligent Robots and Systems (IROS '01) (Hawaii), vol. 1, OctoberNovember 2001, pp. 298-303.

[5] O. Friberg, A set of parameters for finite rotations and translations, Comput. Methods Appl. Mech. Engrg. 66 (1988), 163-171.

[6] H. Hatze, A general myocybernetic control model of skeletal muscle, Biol. Cyber. 28 (1978), no. 3, $143-157$.

[7] J. Hertzberg, How to plan under uncertainty, and use a logical action model, Modelling and planning for sensor based intelligent robot systems (Proc. of Dagstuhl Workshop, October, 1994) (H. Bunke, T. Kanade, and H. Noltemeier, eds.), Machine Perception and Artificial Intelligence, vol. 21, World Scientific, Singapore, 1995, pp. 160-175.

[8] A. V. Hill, The heat of shortening and the dynamic constants of muscle, Proc. Roy. Soc. Ser. B 126 (1938), 136-195.

[9] J. C. Houk, Regulation of stiffness by skeletomotor reflexes, Annu. Rev. Physiol. 41 (1979), 99 114.

[10] J. C. Houk, J. T. Buckingham, and A. G. Barto, Models of the cerebellum and motor learning, Behav. Brain Sci. 19 (1996), no. 3, 368-383.

[11] A. Isidori, Nonlinear Control Systems: An Introduction, 2nd ed., Springer, Berlin, 1989.

[12] T. Ivancevic, L. C. Jain, and M. Bottema, A new two-feature FAM-matrix classifier for breast cancer diagnosis, Proc. 3rd International Conference on Knowledge-Based Intelligent Information Engineering Systems (KES '99), IEEE Press, Adelaide, September 1999, pp. 305-308.

[13] _ A new two-feature GBAM-neurodynamical classifier for breast cancer diagnosis, Proc. 3rd International Conference on Knowledge-Based Intelligent Information Engineering Systems (KES '99), IEEE Press, Adelaide, September 1999, pp. 296-299.

[14] V. Ivancevic, Introduction to Biomechanical Systems: Modeling, Control and Learning, Scientific Book, Belgrade, 1991.

[15]_- Generalized Hamiltonian biodynamics and topology invariants of humanoid robots, Int. J. Math. Math. Sci. 31 (2002), no. 9, 555-565.

[16] Symplectic rotational geometry in human biomechanics, SIAM Rev. 46 (2004), no. 3, $455-474$.

[17] V. Ivancevic and C. E. M. Pearce, Poisson manifolds in generalized Hamiltonian biomechanics, Bull. Austral. Math. Soc. 64 (2001), 515-526.

[18] _ Topological duality in humanoid robot dynamics, ANZIAM J. 43 (2001), no. 2, 183194.

[19] V. Ivancevic and M. Snoswell, Fuzzy-stochastic functor machine for general humanoid-robot dynamics, IEEE Trans. Syst., Man, Cybern. B 31 (2001), no. 3, 319-330.

[20] D. Katic and M. Vukobratovic, A neural network-based classification of environment dynamics models for compliant control of manipulation robots, IEEE Trans. Syst., Man, Cybern. B 28 (1998), no. 1, 58-69.

[21] B. Kosko, Neural Networks and Fuzzy Systems: A Dynamical Systems Approach to Machine Intelligence, Prentice-Hall, New Jersey, 1992. 
[22] C. Mladenova, Mathematical modelling and control of manipulator systems, Int. J. Robotics and Computer-Integrated Manufacturing 8 (1991), no. 4, 233-242.

[23] C. D. Mladenova, Applications of Lie group theory to the modeling and control of multibody systems, Multibody Syst. Dyn. 3 (1999), no. 4, 367-380.

[24] H. Nijmeijer and A. van der Schaft, Nonlinear Dynamical Control Systems, Springer, New York, 1990.

[25] S. Schaal, Is imitation learning the route to humanoid robots?, Trends Cogn. Sci. 3 (1999), no. 6, 233-242.

[26] S. Schaal and C. G. Atkeson, Constructive incremental learning from only local information, Neural Comput. 10 (1998), no. 8, 2047-2084.

[27] H. Schaub, P. Tsiotras, and J. L. Junkins, Principal rotation representations of proper $N \times N$ orthogonal matrices, Internat. J. Engrg. Sci. 33 (1995), no. 15, 2277-2295.

[28] H. Seraji, Configuration control of redundant manipulators: theory and implementation, IEEE Trans. Robot. Automat. 5 (1989), no. 4, 472-490.

[29] M. D. Shuster, A survey of attitude representations, J. Astronaut. Sci. 41 (1993), no. 4, 439-517.

[30] P. van der Smagt (ed.), Self-Learning Robots III Brainstyle Robotics: The Cerebellum Beyond Function Approximation, IEE, London, 1999.

[31] M. Vukobratovic, B. Borovac, D. Surla, and D. Stokic, Biped Locomotion: Dynamics, Stability, Control, and Applications, Springer, Berlin, 1990.

[32] D. E. Whitney, Historical perspective and state of the art in robot force control, Int. J. Robot. Res. 6 (1987), no. 1, 3-14.

[33] D. R. Wilkie, The mechanical properties of muscle, Br. Med. Bull. 12 (1956), no. 3, 177-182.

[34] T. Yoshikawa, Analysis and control of robot manipulators with redundancy, Robotics Research: The First International Symposium (M. Brady and R. Paul, eds.), MIT Press, Massachusetts, 1984, pp. 735-748.

[35] E. Young, Mathematical twist reveals the agony of back pain, New Scientist (2003), August.

Vladimir Ivancevic: Land Operation Division, Defence Science and Technology Organisation, Department of Defence, P.O. Box 1500, Edinburgh 5111, SA, Australia

E-mail address: vladimir.ivancevic@dsto.defence.gov.au

Nicholas Beagley: Land Operation Division, Defence Science and Technology Organisation, Department of Defence, P.O. Box 1500, Edinburgh 5111, SA, Australia

E-mail address: nicholas.beagley@dsto.defence.gov.au 


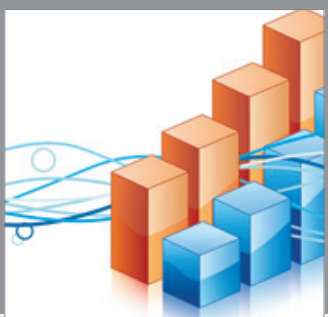

Advances in

Operations Research

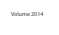

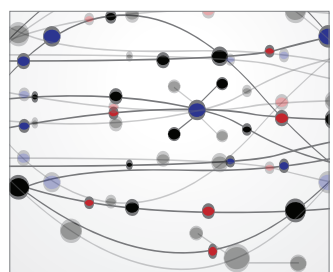

\section{The Scientific} World Journal
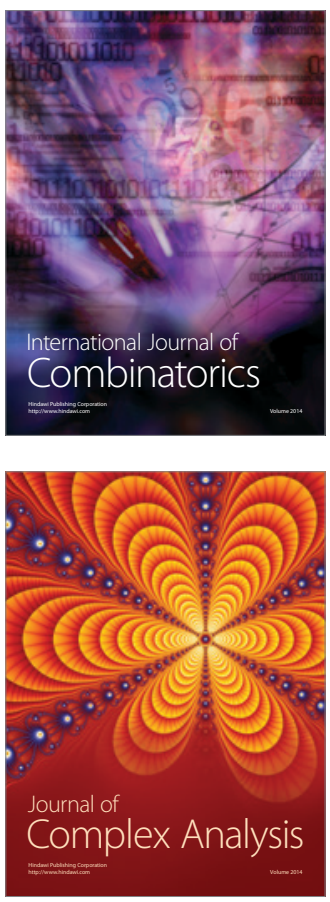

International Journal of

Mathematics and

Mathematical

Sciences
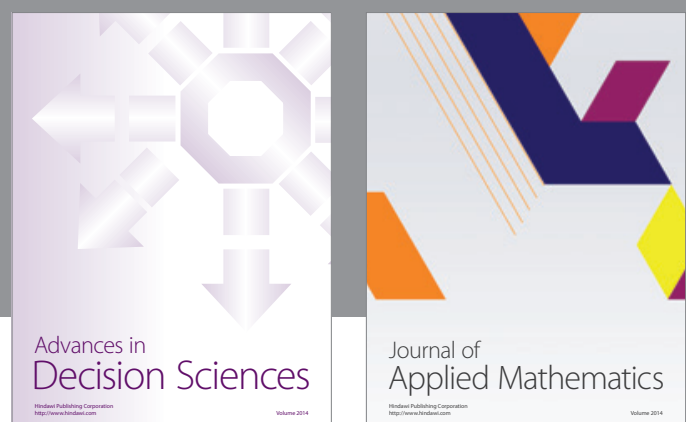

Journal of

Applied Mathematics
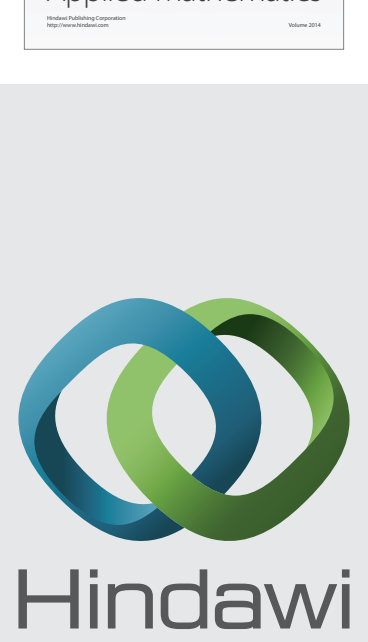

Submit your manuscripts at http://www.hindawi.com
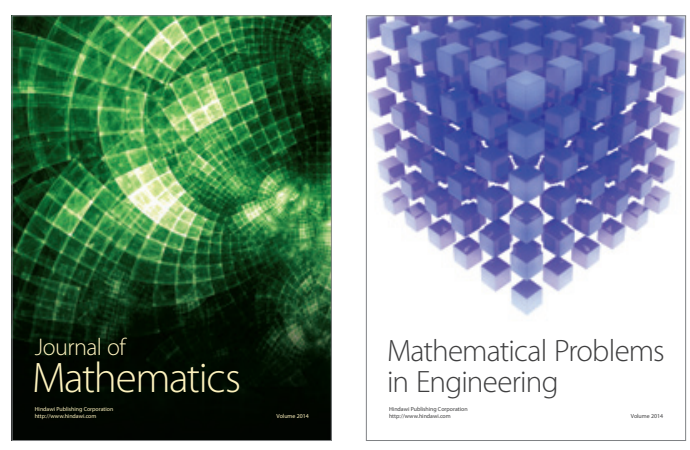

Mathematical Problems in Engineering
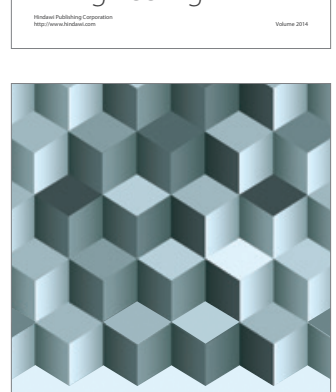

Journal of

Function Spaces
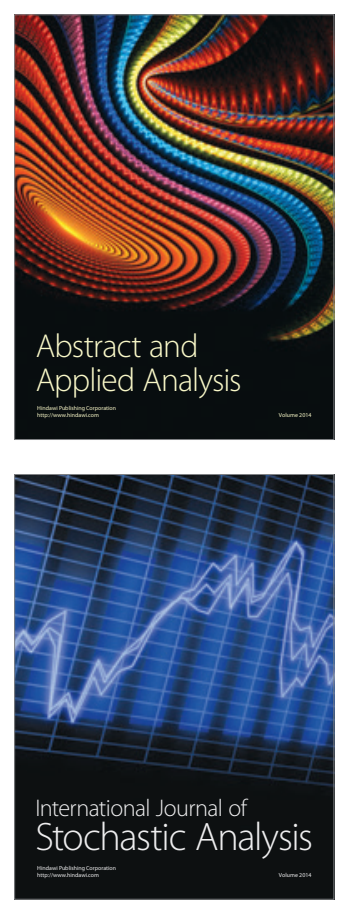

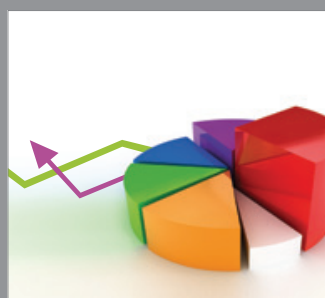

ournal of

Probability and Statistics

Promensencen
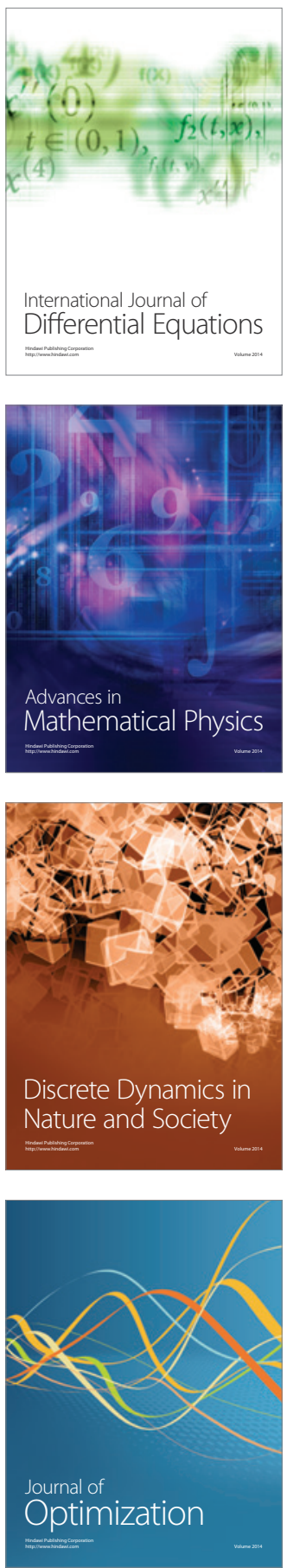